\title{
Acute Bleeding in the Head and Neck: Angiographic Findings and Endovascular Management
}

\author{
L.-B. Zhao, H.B. Shi, S. Park, D.g. Lee, J.H. Shim, D.H. Lee, and D.C. Suh
}

\begin{abstract}
BACKGROUND AND PURPOSE: Life-threatening bleeding in the head and neck requires urgent management. This study evaluated the angiographic findings related to head and neck bleeding and presents endovascular management techniques.
\end{abstract}

MATERIAL AND METHODS: Sixty-one consecutive patients who presented with acute bleeding in the head and neck areas and subsequently underwent endovascular therapy between January 2002 and October 2012 were included in our study. We evaluated the angiographic findings, techniques, and results of endovascular management.

RESULTS: Contrast leakage ( $n=10)$, pseudoaneurysm $(n=20)$, or both $(n=10)$ were the most common life-threatening angiographic findings (66\%) and were the foci of immediate embolization or endoluminal vessel reconstruction. Seventeen patients (28\%) had hypervascular staining of the tumor or mucosa, and 4 patients $(6 \%)$ did not have any abnormal findings. The acute bleeding was successfully controlled by endovascular management according to the bleeding foci. Carotid arterial lesions, so-called "carotid blowout," required reconstructive or deconstructive therapy. Bleeding of the external carotid artery required specific branch embolization by a combination of various embolic materials. No procedure-related complications occurred except in 1 patient who experienced acute infarction caused by thromboemboli from the covered stent. Seventeen patients (28\%) were retreated due to rebleeding after the mean 20-month follow-up.

CONCLUSIONS: Contrast leakage or a pseudoaneurysm or both seen on angiography are active bleeding foci and targets for therapy in patients with acute bleeding in the head and neck area. Despite different bleeding-control strategies according to vessel involvement, endovascular treatment is safe and effective for controlling hemorrhage.

ABBREVIATIONS: $\mathrm{CBS}=$ carotid blowout syndrome; $\mathrm{ECA}=$ external carotid artery

A cute bleeding in the head and neck area occurs due to various causes and often is a life-threatening situation. If conservative management is unsuccessful, conventional angiography followed by endovascular treatment can have a major role in localizing the source of the bleeding and obliterating bleeding foci, thus leading to immediate and complete hemostasis.

Head and neck cancers are the main cause of intractable hemorrhage from local tumor irradiation or spontaneous tumor bleeding. ${ }^{1-5}$ Acute rupture of irradiated, large vessels is a rare but

Received March 14, 2013; accepted after revision May 20.

From the Departments of Radiology and Research Institute of Radiology (L.-B.Z. H.B.S., S.P., D.-g.L., J.H.S., D.H.L., D.C.S.), University of Ulsan, College of Medicine, Asan Medical Center, Seoul, Korea; and Department of Radiology (L.-B.Z., H.B.S.), First Affiliated Hospital of Nanjing Medical University, Nanjing, Nanjing, China. H.B.S. and D.C.S. contributed equally as corresponding authors.

Please address correspondence to Dae Chul Suh, MD, PhD, Department of Radiology, Asan Medical Center, University of Ulsan, College of Medicine, 86 Asanbyeongwon-Gil, Songpa-Gu, Seoul 138-736, Korea; e-mail: dcsuh@amc.seoul.kr

Indicates article with supplemental on-line table.

http://dx.doi.org/10.3174/ajnr.A3761 life-threatening therapy complication. ${ }^{6}$ In addition to tumor-related bleeding, there is also iatrogenic bleeding related to surgical procedures or craniomaxillofacial trauma, which can also lead to intractable, life-threatening bleeding.

The distribution of bleeding foci is diverse and can range from large vessels, such as the internal or common carotid arteries, to small branches of the external carotid artery (ECA). Identification of the lesion location is mandatory when performing an angiographic procedure and analyzing the angiographic findings because the exact localization of the bleeding site is associated with immediate bleeding control.

The endovascular management of bleeding arising from the extracranial carotid arteries, the so-called "carotid blowout syndrome," has been extensively reported. ${ }^{7-14}$ However, to our knowledge, the exact extent of the carotid blowout syndrome (CBS) in patients with acute bleeding has not been evaluated or compared with that of patients without CBS, despite the completely different application of endovascular hemostatic techniques for CBS versus non-CBS lesions. Therefore, we present our 

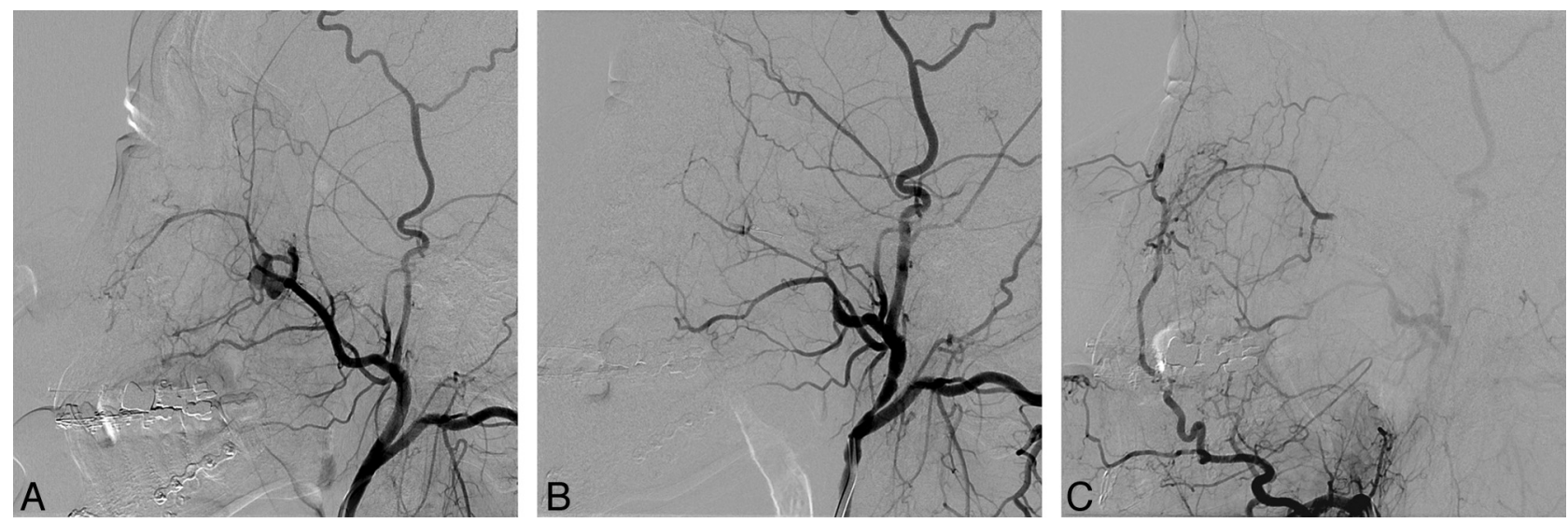

FIG 1. A 24-year-old male patient who presented with intractable epistaxis after sagittal split ramus osteotomy. A, Lateral left ECA arteriogram shows a pseudoaneurysm at the internal maxillary artery segment at the pterygopalatine fossa. $B, A$ microcatheter was advanced to the distal maxillary artery beyond the pseudoaneurysm that was embolized by using a coil. C, Selective injection of the left facial artery shows collaterals from the ascending artery to the distal maxillary artery via the infraorbital artery. Note the absence of filling of the pseudoaneurysm from retrograde flow of the distal maxillary artery.

experience of endovascular management of 61 patients with acute head and neck bleeding from a variety of causes, with an emphasis on the angiographic results and the subsequent endovascular management according to the angiographic findings. We also compared the differences between patients with and without tumors related to the causative angiographic features, treatment outcome, and rate of endovascular retreatment.

\section{MATERIALS AND METHODS}

\section{Patients}

Sixty-one consecutive patients (49 men and 12 women; 17-79 years of age [mean age, 53 years]) who presented with acute hemorrhage in the head and neck and were subsequently treated by endovascular therapy between January 2002 and October 2012 were included from the neurointerventional data base of our institution. Three bleeding sites were classified according to the lesion location with or without an open wound in the mucosa or skin: bleeding or hematoma in the oral cavity $(n=32)$, nasal cavity $(n=20)$, or head and neck area $(n=9)$. The estimated causes of bleeding were head and neck cancers $(n=36)$ associated with prior radiation treatment $(n=33)$ or tumor bleeding itself $(n=3)$. Non-tumor-related bleeding $(n=25)$ included iatrogenic complications $(n=13)$, trauma $(n=5)$, refractory idiopathic epistaxis $(n=3)$, intraosseous mandibular arteriovenous malformation $(n=1)$, invasive fungal sinusitis $(n=1)$, a history of antiplatelet medication $(n=1)$, or history of liver transplantation $(n=1)$. Iatrogenic bleeding $(n=13)$ included postsurgery $(n=8)$, postbiopsy $(n=4)$, and post-C-line insertion $(n=1)$. Regarding the 8 patients with postsurgical bleeding, in 3 , it occurred after maxillofacial plastic surgery; in 3, after endoscopic sinus surgery; in 1, after posterior cervical fusion; and in 1, after wide excision of recurrent left tonsillar cancer.

There was no commercial involvement in the design, conduct, or analyses of this study. The institutional review board of the Asan Medical Center approved both the study design and use of clinical data, and all patients provided written informed consent.

\section{Procedures}

All procedures were performed with the patient under local anesthesia. High-resolution digital fluoroscopy with biplane road-mapping capability and subtraction techniques was used. Angiography usually began by using a $4 \mathrm{~F}$ angiocatheter with a 0.035 -inch inner diameter, which can also be used as a guiding catheter for a microcatheter. If further procedures using a covered stent were required, a $9 \mathrm{~F}$ guiding catheter was introduced.

Bilateral selective angiography of the common, internal, and external carotid arteries was performed. Regarding patients with neck bleeding or hematoma, both sides of the thyrocervical or costocervical trunks were also investigated. If the patients presented with massive bleeding or hemodynamic instability, the most likely injured vessels were first evaluated to manage the bleeding immediately, and the remaining vessels were studied if indicated.

Angiographic findings of pathologic vascular lesions were recorded as being caused by contrast leakage, pseudoaneurysms combined with contrast leakage, pseudoaneurysms, fistula, tumor, or hypervascular mucosal staining or as negative (without abnormal findings). Lesion locations were recorded as branches of the ECA, the carotid arteries including the internal carotid artery, the common carotid artery, and the carotid artery bulb or the thyrocervical or costocervical trunks.

If bleeding was localized to the ECA or its branches, they were occluded by using polyvinyl alcohol particles (150 250 $\mu \mathrm{m})$, Gelfoam (Phadia, Uppsala, Sweden), glue, fibered platinum or detachable coils, or a combination of these embolic materials. Collateral cerebral circulation via the circle of Willis, leptomeningeal vessels, or the ophthalmic artery was evaluated by using compression angiographic studies if bleeding originated from the internal or common carotid artery. The covered nitinol stent (NITI-S Stent; Taewoong Medical, Seoul, Korea), composed of a self-expanding nitinol wire covered with polytetrafluoroethylene, was used in patients with incomplete collaterals determined on a compression test performed during the procedure or if there was 

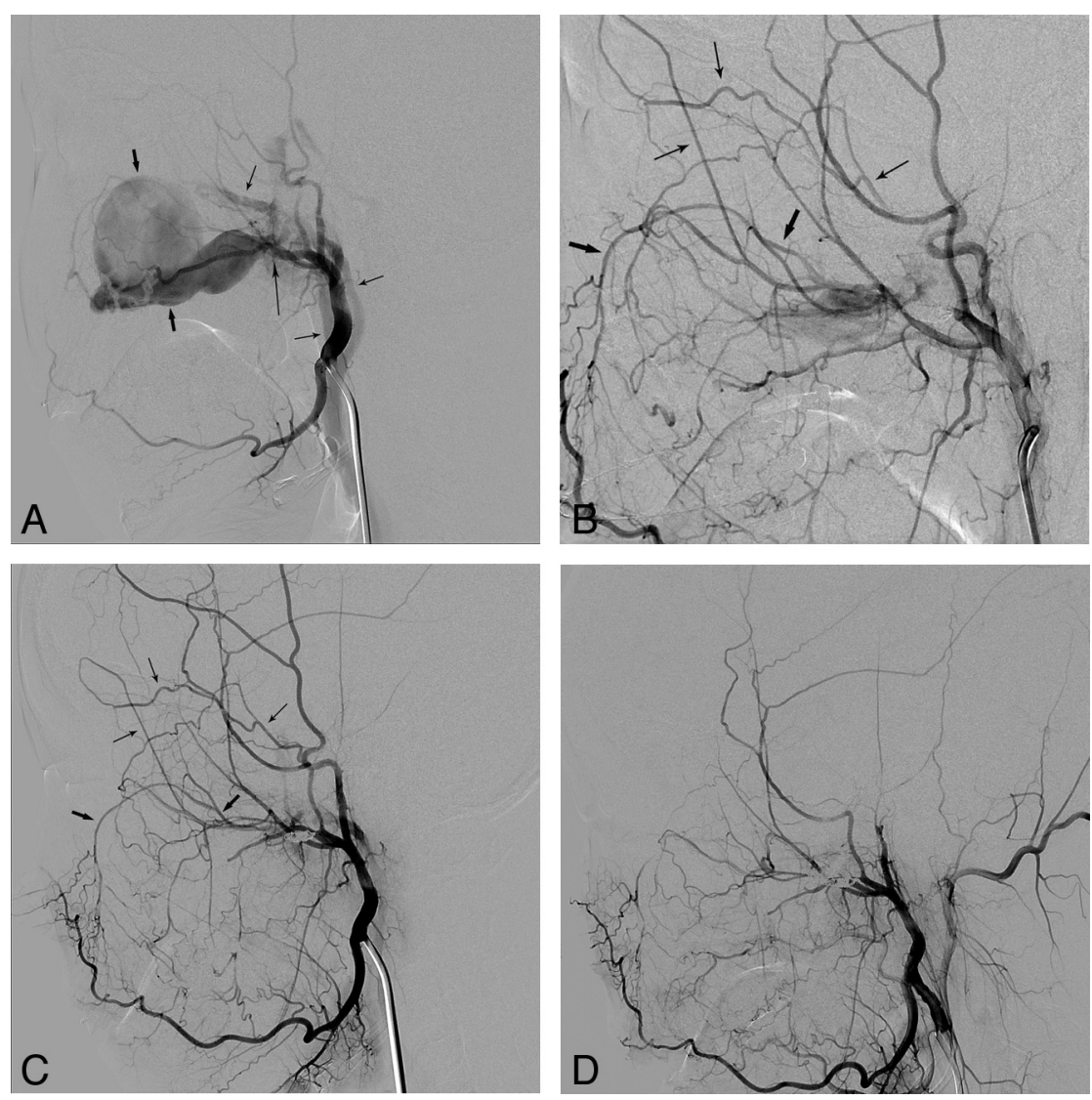

FIG 2. A 19-year-old female patient who presented with intractable epistaxis after maxillofacial plastic surgery. A, An external carotid angiogram shows a large pseudoaneurysm (short thick arrows) and a fistula (long thin arrow) with an arteriovenous shunt. Note the early filling veins (short thin arrows). B, An angiogram obtained following coil embolization of the maxillary artery at the aneurysm site shows retrograde filling of the remaining shunt through the infraorbital artery (short thick arrows) via the facial artery and the zygomatico-orbital branch (short thin arrows) of the superficial temporal artery. C, An angiogram obtained the following day revealed a slightly smaller but still remaining fistula with persistent bleeding. Note the retrograde filling as indicated on $B$ by the same arrows. $D$, The bleeding was finally controlled after additional coil embolization via the zygomatico-orbital branch (short thin arrows, $B$ ) into the fistula.

no time to perform occlusion or a compression test due to unstable vital signs due to massive bleeding. ${ }^{7}$ The diameters and lengths of the stents were $10 \times 70 \mathrm{~mm}$ or $10 \times 50 \mathrm{~mm}$. Because the stent adheres to the polytetrafluoroethylene graft by using polyurethane, it is composed of polyurethane on its outer surface and the polytetrafluoroethylene sheet on its inner surface. ${ }^{8}$ Antiplatelet medication with $200 \mathrm{mg}$ of aspirin and a loading dose of $300 \mathrm{mg}$ of clopidogrel was administered immediately after stent placement if patients were not already taking these medications. After the revascularization procedure, maintenance doses of $100 \mathrm{mg}$ of aspirin and $75 \mathrm{mg}$ of clopidogrel were given; $75 \mathrm{mg}$ of clopidogrel for at least 6 months and $100 \mathrm{mg}$ of aspirin as a permanent medication were also given. The common carotid artery or ICA was completely occluded in patients with uncontrolled massive carotid hemorrhage if good collateral cerebral circulation was seen on the compression test.

We collected data on the procedural and clinical outcomes, complications resulting from the procedures, additional repeated treatments, and survival data from the patients' medical records.

\section{RESULTS}

\section{Angiographic Findings}

The On-line Table summarizes the patients' angiographic findings and the baseline characteristics. Forty patients $(66 \%)$ presented with bleeding signs at the time of their angiographic procedure, including 20 cases of pseudoaneurysm (Fig 1), 10 cases of contrast leakage, and 10 cases of pseudoaneurysm combined with contrast leakage. A fistula was present in a patient with a large pseudoaneurysm caused by postoperative injury of the maxillary artery in the pterygopalatine fossa (Fig 2). No bleeding sign was seen in 21 patients (34\%), including 17 with hypervascular tumor or mucosal staining (28\%) and 4 with negative findings $(6 \%)$. Angiographic bleeding foci were identified as CBS in $25 \%(15 / 61)$ and non-CBS in 75\% (46/ $61)$ of our patients. CBS involved the internal $(n=6)$ or common $(n=8)$ carotid artery or the carotid artery bulb $(n=1)$. There was non-CBS in the ECAs in 40 patients, in the thyrocervical trunks in 2 patients, and in 4 patients with negative angiographic findings. Thirty-six patients $(36 / 61,59 \%)$ were identified as having tumor-related bleeding versus 25 patients $(25 / 61,41 \%)$ with non-tumor-related bleeding.

\section{Procedural Outcome}

Forty-six patients underwent transarterial embolization treatment: The lesions were located in the ECAs in 40 patients, at the thyrocervical trunks in 2 , and negative angiographic findings existed in 4 patients. For these patients with negative findings, embolization of the ipsilateral ECA branches with polyvinyl alcohol particles on the bleeding side was performed in 3 for prevention of rebleeding, while the other patient was embolized on both sides of the ECA branches. As to the 15 patients with bleeding lesions located on the ICA, common carotid artery, or bulb, 10 underwent covered stent insertion and the other 5 underwent ICA or common carotid artery occlusion by embolization.

\section{Clinical Outcome and Recurrent Bleeding}

Initial hemostasis was achieved in all patients. There were 17 $(28 \%, 17 / 61)$ patients who underwent repeated endovascular management due to rebleeding. Seven of these patients experienced recurrent bleeding during the same hospital stay; 4 had rebleeding in the same vascular locations, and 3 , in different locations. Five of these 7 patients had head and neck cancers, and 3 presented with CBS. Ten patients were readmitted due to rebleeding after discharge (Fig 3), and they subsequently underwent endovascular retreatment at a mean interval of 2 months (range, 

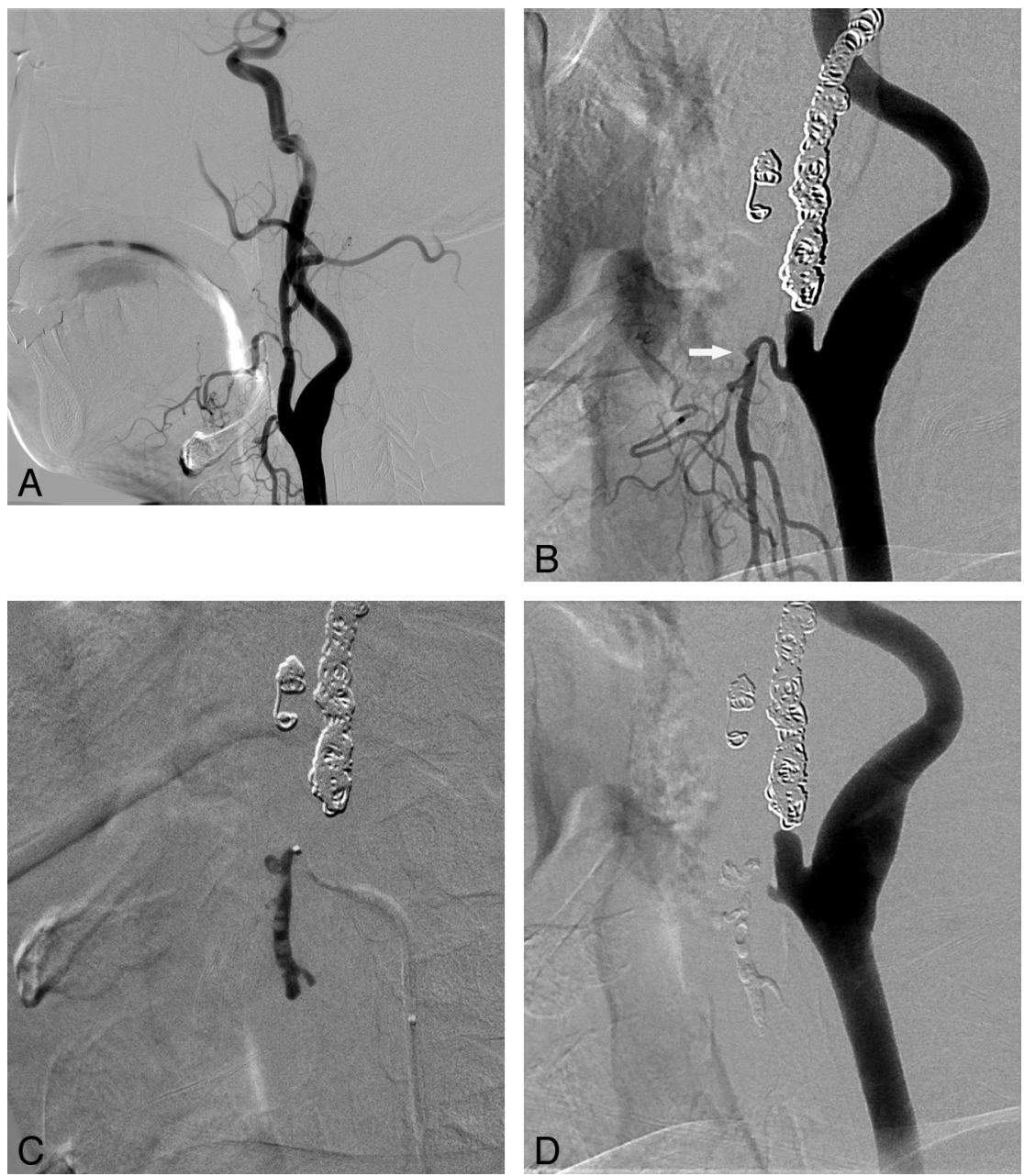

FIG 3. Endovascular treatment for recurrent bleeding approximately 2 months and 15 days after the initial embolization of the left ECA in a 45-year-old male patient with tonsil cancer. A, At the time of the first bleeding, the right facial and nearby maxillary arteries showing irregularities were regarded as the origin of the initial bleeding site and were embolized with coils as shown in $B$. $B$, Left common carotid arteriogram obtained at the time of the second bleeding reveals a tiny pseudoaneurysm (white arrow) in the proximal segment of the left superior thyroidal artery. Note the coils used in the previous embolization. C, Glue was used for embolization of the left superior thyroidal artery, and the final angiogram shows no residual pseudoaneurysm filling. The patient's bleeding was controlled thereafter.

1-6 months) after their initial endovascular treatment: 8 patients had head and neck cancers ( 5 of these 8 patients presented with CBS), 1 had juvenile angiofibroma, and 1 had an intraosseous mandibular arteriovenous malformation.

\section{Complications and Follow-Up}

One patient experienced occlusion of the ipsilateral middle cerebral artery branches during the covered stent placement and acute infarction in the corresponding vascular territory, probably due to no premedication with an antiplatelet agent. This patient presented with massive neck bleeding and had a history of radiation therapy for supraglottic cancer. The bleeding originated from rupture of the exposed carotid artery through the ulcerated mucosa. When he was brought to the angiography suite with manual compression of the bleeding site, his vital signs were unstable due to the uncontrollable massive bleeding, despite an urgent transfusion. The angiogram showed a common carotid artery blowout with massive, active, contrast leakage. Covered stent placement was immediately introduced to control the active bleeding. An angiogram obtained after stent-graft placement showed successful hemostasis and multiple embolic occlusions of the MCA branches. MR imaging performed the following day showed acute right MCA territorial infarction. The patient's neurologic status subsequently improved and became stable (modified Rankin Scale $=2$ ) for 17 months; then the patient was lost to follow-up (Fig 4).

Delayed cerebral infarcts were present 3 or 4 months after endovascular treatment, respectively, in 2 patients who underwent stent placement for CBS. The deficit seen in the first patient resulted from embolization or ligation of the internal carotid artery performed to control recurrent CBS after stent placement. In the second patient, it resulted from occlusion of the covered stent or local progression of nasopharyngeal carcinoma. No neurologic deficits were found in the patients who initially underwent obliteration of the entire involved carotid artery by using coil embolization.

Extrusion of the coils previously placed for embolization was observed in 4 patients in whom the carotid arteries had been exposed to the infected wound cavity. These patients subsequently underwent ligation $(n=3)$ or re-embolization $(n=1)$ of the involved carotid artery. No brain abscess developed in our series, and no complications were seen in patients who underwent embolization of the ECA or its branches.

Of the 36 patients with head and neck cancers, 8 did not experience any further bleeding events during the mean follow-up time of 27 months (range, 12-59 months). Twenty-four of these patients with cancer died due to recurrent or progressive tumor growth-related problems, with a mean survival time of 3 months (range, 1-10 months). As for the other 4 patients with cancer, 3 were lost to follow-up 1 month after the initial endovascular treatment, and the other was lost after 3 months.

Among the 10 patients who presented with CBS and underwent covered stent placement, 7 died within 1-10 months (median, 2 months). The exact cause of death could not be determined in these patients because they were regarded as having terminal-stage cancer. Two patients were lost to follow-up after 1 month. One patient with an infarct in the right middle cerebral artery territory was followed for 17 months (Fig 4).

Two patients without tumor died of traumatic intracranial hemorrhage: One patient died from a car collision 1 week follow- 

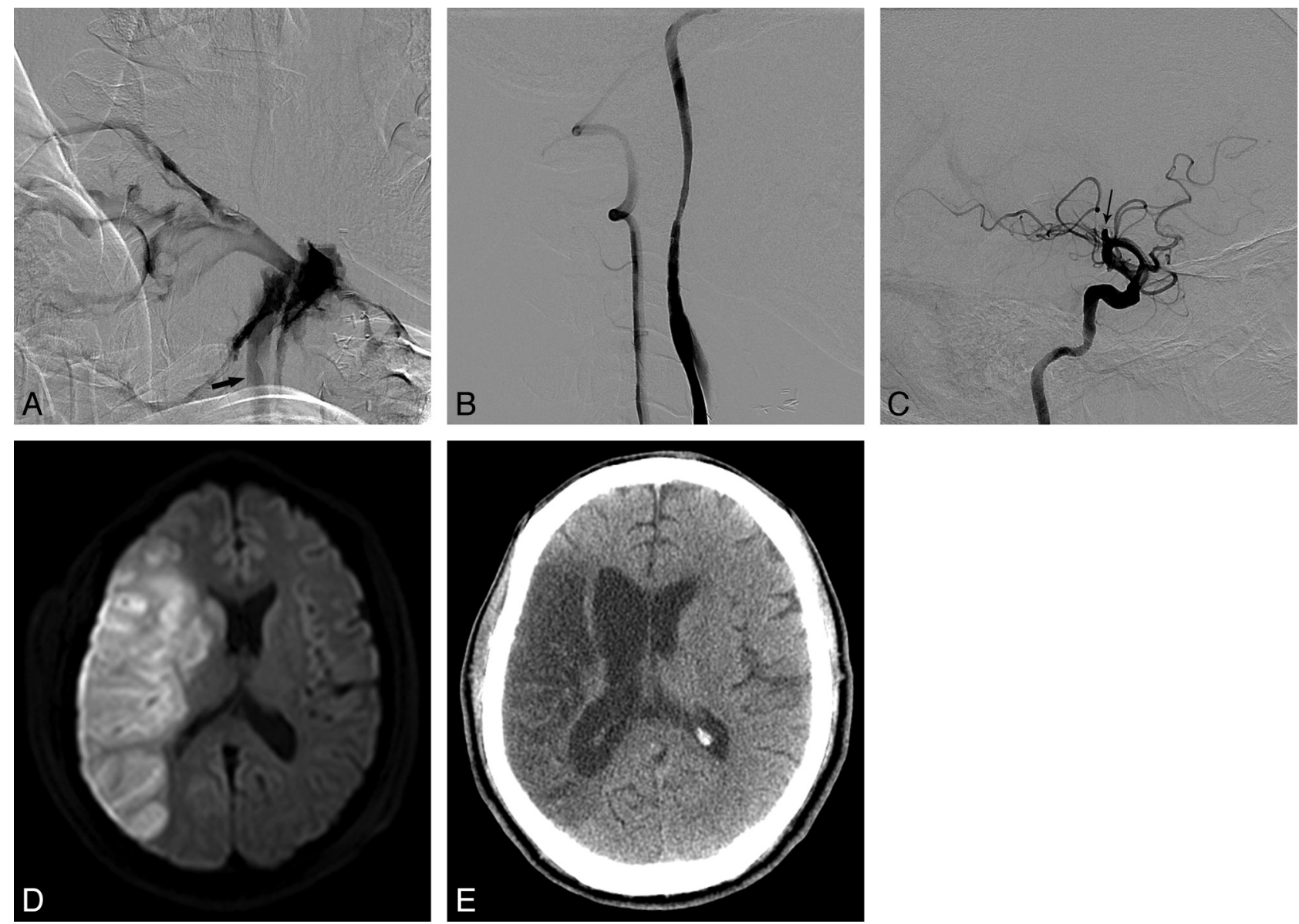

FIG 4. A 51-year-old male patient with a history of supraglottic cancer who presented with massive bleeding at the ulcerative wound site in the right neck. After tracheostomy, he became drowsy and developed left-sided weakness in both the upper and lower extremities. $A$, A common carotid arteriogram shows extensive contrast leakage due to massive bleeding. No filling of the internal carotid artery from the common carotid artery (arrow) was seen. B, Bleeding was stopped following deployment of a covered stent. C, There is an occlusion of the right middle cerebral artery at the proximal M2 segment (arrow). D, Diffusion-weighted image obtained the next day shows a territorial infarct in the right MCA territory. E, CT scan obtained 17 months later shows the old infarction in the right middle cerebral artery territory, though the patient's weakness had improved and he walked without assistance.

ing endovascular treatment, and the other died of an event related to liver transplantation 7 months after embolization.

\section{DISCUSSION}

Our study revealed that angiographically detected active bleeding signs, such as contrast leakage and/or pseudoaneurysm requiring urgent endovascular treatment, were noted in $66 \%$ of our patients. As to the patients in whom a pseudoaneurysm was only identified at the time of the angiography, management similar to that of the patients with active bleeding was also required because the risk of delayed or repetitive massive hemorrhage is very high, even though these patients may not present with active bleeding when brought to the angiography suite. Nonselective particle embolization in the external carotid arterial branches was sufficient as a preventive measure in those patients who showed only hypervascular staining (28\%) or normal angiographic findings (6\%).

When we categorized our patients as CBS versus non-CBS according to the lesion involvement, 25\% were CBS and immediately required either reconstructive or deconstructive management of the carotid artery to control life-threatening bleeding, as we previously reported. ${ }^{7-9}$ Non-CBS, in $75 \%$ of our patients, included those with lesions located at the branches of the ECA or the thyrocervical trunks. An active angiographic bleeding sign of contrast leakage and/or a pseudoaneurysm was seen in $56 \%$ of patients who presented with non-CBS, thus indicating direct injury to the arterial branches. When one treats injured vessels as a bleeding focus in branches of the ECA, the stumps proximal and distal to the lesions should be identified and embolized together, as shown in Fig 1. For example, when there is a pseudoaneurysm or fistula in the maxillary artery, the distal maxillary artery beyond the bleeding focus of the injured vessel should be identified by selective angiography of the ipsilateral facial artery. If only the proximal segment is embolized, as shown in Fig 2, a collateral to the distal maxillary artery, such as the facial artery, can flow into the injured vessel in retrograde fashion. Therefore, a collateral through the other side of a stump may cause bleeding recurrence due to incomplete embolization.

When we divided our patients into tumor-versus-nontumor groups, we found that head and neck cancer was the main cause of acute bleeding in our series and was present in 59\% of the patients. Previous radiation therapy, recurrent carcinoma, and surgical complications such as infection and fistula formation may all contribute to the onset of such bleeding and may make 
controlling it more difficult. ${ }^{13,15,16}$ Repeated bleeding in such patients is also a common and troublesome problem. ${ }^{17}$ In our series, the rate of endovascular retreatment for rebleeding in patients with cancers $(36 \%)$ was higher compared with that for patients without tumor (16\%), which can be attributed to carcinoma recurrence, radiation therapy, or surgical complications (Fig 3).

Iatrogenic acute bleeding was found in 21\% (13 patients) of our patients and included those who had undergone surgery $(n=$ 8 ), biopsy $(n=4)$, and C-line-insertion-associated bleeding complications $(n=1)$. Among the 8 patients who presented with uncontrolled oronasal hemorrhage after surgeries, 5 showed active contrast leakage or pseudoaneurysm on angiography. Maxillofacial surgical procedures, such as orthognathic surgery, may be associated with significant blood loss and, infrequently, lifethreatening hemorrhage caused by injury to the vascular structures in the pterygopalatine fossa, such as the pterygoid venous plexus, the descending palatine arteries, the sphenopalatine arteries, or the maxillary artery itself, leading to acute epistaxis. ${ }^{18}$ Pooled data revealed that in $72 \%$ of the Le Fort osteotomies, an arterial source was the culprit, whereas in almost $19 \%$ of the cases, the source of hemorrhage was venous. In the remaining cases, the exact source was unidentifiable. In mandibular sagittal split osteotomies, an arterial source was the cause of hemorrhage in $48.2 \%$, and a venous source, in $20.6 \%$ of the cases. ${ }^{19}$ In cases in which damage to the inferior alveolar artery, internal maxillary artery, or facial artery was suspected, blood loss ranged from 1500 to $3000 \mathrm{~mL} .{ }^{20}$ In these cases, obvious bleeding signs will be more likely on angiograms compared with those with lesions located in veins or with unidentifiable sources.

There were 5 trauma patients ( $8 \%)$ in our series, and all presented with massive bleeding. Trauma is the ninth leading cause of death worldwide, and traumatic injury of a vessel is a relatively common occurrence in the emergency department. ${ }^{21}$ With injury caused by either blunt or penetrating forces, these patients presented with acute bleeding due to laceration or fistula of vessels as a result of penetrating injury or bone fractures. For these patients, anterior and posterior nasal packing as the initial measure used to manage massive oronasal hemorrhage is supported by the published medical literature. ${ }^{22}$ Other conventional techniques, such as cauterization or ligation of the ECA and reduction and fixation of a facial fracture, can secure hemostasis in most remaining cases. Infrequently, the bleeding may become intractable and thus lifethreatening. ${ }^{23,24}$ If conventional techniques fail to stop the bleeding, transarterial embolization of relevant vessels may be effective as it was in our patients in whom all the bleeding foci were identified at the branches of the ECA, which were successfully embolized without rebleeding or complications after transarterial embolization.

Although endovascular management for acute head and neck bleeding has been extensively reported, especially regarding the management of CBS and bleeding with head and neck cancers, little emphasis has been placed on studying the angiographic findings or on the differences in treatment outcome and the rate of endovascular retreatment between patients with and without tumors. In this study, we present our 11 years of clinical experience with endovascular management of head and neck acute bleeding caused by various pathologic etiologies. We studied the angio- graphic findings and compared the differences in the angiographic findings, endovascular techniques, clinical outcomes, and the rate of endovascular retreatment among patients with and without tumors.

There are several limitations to our study. Because our series was a retrospective analysis, comparative application of different endovascular methodologies used to control the bleeding was not tested. Even though our series is rather large, the incidence and comparison of CBS versus non-CBS or tumor-related versus nontumor-related bleeding can be more accurately evaluated or compared by performing a multicenter registry.

\section{CONCLUSIONS}

Acute bleeding in the head and neck is a common life-threatening situation. If conventional techniques fail to stop the hemorrhaging, conventional angiography can have an important role in localizing the bleeding foci and controlling the bleeding by endovascular treatment, which is a safe and effective therapy for the management of hemorrhage.

\section{REFERENCES}

1. Pereira J, Phan T. Management of bleeding in patients with advanced cancer. Oncologist 2004;9:561-70

2. Dhiwakar M, Khan NA, McClymont LG. Surgical resection of cutaneous head and neck lesions: does aspirin use increase hemorrhagic risk? Arch Otolaryngol Head Neck Surg 2006;132:1237-41

3. Bachar G, Esmat N, Stern S, et al. Transarterial embolization for acute head and neck bleeding: eight-year experience with emphasis on rebleeding risk in cancer patients. Laryngoscope 2013;123:1220-26

4. Sesterhenn AM, Iwinska-Zelder J, Dalchow CV, et al. Acute haemorrhage in patients with advanced head and neck cancer: value of endovascular therapy as palliative treatment option. J Laryngol Otol 2006;120:117-24

5. Remonda L, Schroth G, Caversaccio M, et al. Endovascular treatment of acute and subacute hemorrhage in the head and neck. Arch Otolaryngol Head Neck Surg 2000;126:1255-62

6. Toyoda T, Sawatari K, Yamada T, et al. Endovascular therapeutic occlusion following bilateral carotid artery bypass for radiationinduced carotid artery blowout: case report. Radiat Med 2000; 18:315-17

7. Pyun HW, Lee DH, Yoo HM, et al. Placement of covered stents for carotid blowout in patients with head and neck cancer: follow-up results after rescue treatments. AJNR Am J Neuroradiol 2007; 28:1594-98

8. Kim HS, Lee DH, Kim HJ, et al. Life-threatening common carotid artery blowout: rescue treatment with a newly designed self-expanding covered nitinol stent. Br J Radiol 2006;79:226-31

9. Roh JL, Suh DC, Kim MR, et al. Endovascular management of carotid blowout syndrome in patients with head and neck cancers. Oral Oncol 2008;44:844-50

10. Chang FC, Lirng JF, Luo CB, et al. Patients with head and neck cancers and associated postirradiated carotid blowout syndrome: endovascular therapeutic methods and outcomes. J Vasc Surg 2008; 47:936-45

11. Chang FC, Lirng JF, Luo CB, et al. Carotid blowout syndrome in patients with head-and-neck cancers: reconstructive management by self-expandable stent-grafts. AJNR Am J Neuroradiol 2007;28: $181-88$

12. Warren FM, Cohen JI, Nesbit GM, et al. Management of carotid 'blowout' with endovascular stent grafts. Laryngoscope 2002;112: 428-33

13. Chaloupka JC, Putman CM, Citardi MJ, et al. Endovascular therapy for the carotid blowout syndrome in head and neck surgical

AJNR Am J Neuroradiol 35:360-66 Feb 2014 www.ajnr.org 
patients: diagnostic and managerial considerations. AJNR Am J Neuroradiol 1996;17:843-52

14. Ketcham AS, Hoye RC. Spontaneous carotid artery hemorrhage after head and neck surgery. Am J Surg 1965;110:649-55

15. Wong SJ, Machtay M, Li Y. Locally recurrent, previously irradiated head and neck cancer: concurrent re-irradiation and chemotherapy, or chemotherapy alone? J Clin Oncol 2006;24:2653-58

16. Macdonald S, Gan J, McKay AJ, et al. Endovascular treatment of acute carotid blow-out syndrome. J Vasc Interv Radiol 2000;11: $1184-88$

17. Chen YF, Lo YC, Lin WC, et al. Transarterial embolization for control of bleeding in patients with head and neck cancer. Otolaryngol Head Neck Surg 2010;142:90-94

18. Lanigan DT, Hey JH, West RA. Major vascular complications of orthognathic surgery: hemorrhage associated with Le Fort I osteotomies. J Oral Maxillofac Surg 1990;48:561-73

19. Khanna S, Dagum AB. A critical review of the literature and an evi- dence-based approach for life-threatening hemorrhage in maxillofacial surgery. Ann Plast Surg 2012;69:474-78

20. Lanigan DT, Hey J, West RA. Hemorrhage following mandibular osteotomies: a report of 21 cases. J Oral Maxillofac Surg 1991;49: $713-24$

21. Radvany MG, Gailloud P. Endovascular management of neurovascular arterial injuries in the face and neck. Semin Intervent Radiol 2010;27:44-54

22. Cogbill TH, Cothren CC, Ahearn MK, et al. Management of maxillofacial injuries with severe oronasal hemorrhage: a multicenter perspective. J Trauma 2008;65:994-99

23. Ardekian L, Samet N, Shoshani Y, et al. Life-threatening bleeding following maxillofacial trauma. J Craniomaxillofac Surg 1993;21: $336-38$

24. Tung TC, Tseng WS, Chen CT, et al. Acute life-threatening injuries in facial fracture patients: a review of 1,025 patients. J Trauma 2000; 49:420-24 